\title{
Catalytic activity and accessibility of acidic ion-exchange resins in liquid phase etherification reactions
}

\author{
J.H. Badia, C. Fité*, R. Bringué, M. Iborra, F. Cunill \\ Chemical Engineering Department, Faculty of Chemistry, University of Barcelona, C/ Martí i Franquès \\ 1-11 08028, Barcelona, Spain
}

*Corresponding author. Tel.: +34 93402 1304; fax: +34 934021291 E-mail address: fite@ub.edu

\begin{abstract}
Although macroreticular acidic ion-exchange resins have been widely used as catalysts in the industrial world for decades, their catalytic behavior is still far from being completely understood at a molecular level. Several characterization techniques coexist, which provide information about their properties. Only few of these techniques give an actual picture of their working-state features when swollen in anhydrous polar reactive media such as in etherification processes, where they are extensively used. The inverse steric exclusion chromatography technique, based on modeling the micropores structure, or gel-phase, as a set of discrete volume fractions with a characteristic polymer chain density, constitutes an appropriate procedure to assess the morphology of ion-exchangers in the swollen state. Present work proposes an empirical model to correlate the properties of the volume fractions with their catalytic activity in the etherification reaction rates of isobutene by addition of C1 to C4 linear primary alcohols. Sixteen different macroreticular acidic ion-exchange catalysts, both commercial and lab-made, have been used, which differ in acid capacity, sulfonation type, cross-linking degree and swollen-phase volume fractions distribution. Experimental reaction rates have been expressed as a sum of contributions of each individual volume fraction. The contribution of each polymer volume fraction corresponds to the product of the catalyst acidity, the characteristic volume fraction within the gel-phase of the catalyst, and a specific turnover frequency (TOF) of that fraction. Accessibility of the reacting alcohol, expressed in terms of the Ogston coefficient, has been also included in the empirical dependency equation presented in this work.
\end{abstract}

Keywords macroreticular cationic ion-exchange catalysts, gel-phase, etherification

\section{Introduction}

The role of catalysis in the modern industrial chemistry is crucial. Most industrial catalysts are highsurface-area solids onto which active centers are located. These materials have become today critical to mediate the pathways by which chemical reactions occur, enabling highly selective formation of desired products at rates that are commercially viable [1]. Ion-exchange resins are among the polymer-based catalysts, since active sites are supported on the polymer backbone.

They can be classified into two main morphological types: gel-type and macroreticular. Gel-type ionexchangers consist in resins without an appreciable porosity in the dry state, whose interior is accessible only after swelling due to the reaction environment. Macroreticular resins count with agglomerates of gel-type zones, with their respective micropores, that form permanent macropores between them [2].

The most frequent macromolecular structure in this field is polystyrene (PS) crosslinked by divinylbenzene (DVB), typically resulting in spherical beads. DVB percentage plays a key role for the resulting physical properties of a catalyst. For instance, low crosslinked catalysts can swell up to five times their original dry-state volume, what may give rise to drop-pressure problems when used as packing for reactive columns or tubular reactors, whereas highly crosslinked resin networks may result in inconveniently dense, and thus inaccessible, domains. Such domains would become difficult to functionalize in the resin manufacture and they could become hardly accessible to reactants in its catalytic use [3]. 
Regarding the process of functionalization in the resin manufacture, the simplest reaction is the direct sulfonation of polystyrene with concentrated sulfuric acid that gives the ion-exchangers known since the 1950s, though other functional groups can be introduced upon starting from lithiated or chloromethylated polystyrenes [2].

Although nowadays resins are widely used as catalysts in the chemical industry, the understanding of these materials at a molecular scale level has not yet been successfully achieved. For such purpose, an ever-growing set of characterization techniques exists that give light in different ways to the morphological nature of polymeric catalysts. Both classical and modern most relevant physical characterization techniques can be listed as follows: temperature programmed techniques (including thermogravimetry), mass spectrometry, physical adsorption (BET model for determination of specific surfaces), porosimetry (mercury-based measurement of the pore size and size distribution), X-ray technologies, electron microscopy, infrared and Raman spectroscopies, electron spin resonance and magic angle spinning nuclear magnetic resonance [3]. However, none of the listed techniques takes into consideration the micropores generated by the swelling of the polymer skeleton in contact with a liquid. Therefore, one of the most determining aspects in evaluating the interaction of the catalytic behavior of an ion-exchanger with the reacting medium remains unmonitored. As it is known, certain properties of a reaction medium, such as polarity, have been marked to be strongly related to the swelling of the catalysts, allowing a higher accessibility of reactants to the functional groups and, thus, influencing the catalytic activity. Furthermore, the polarity of a reaction medium may vary throughout a reactive process and so would do the catalyst swelling $[2,4,5]$.

Up to date, the only procedure employed to characterize the morphology of ion-exchangers in the swollen state is the Inverse Steric Exclusion Chromatography (ISEC), based on modeling the microporous structure as a set of discrete volume fractions with different characteristic polymer chain densities [6, 7]. The ISEC technique is appropriate to assess the catalytic behavior in chemical reactions, since it is based upon the swelling of ion-exchangers in water, for water was found to be the most suitable solvent in terms of reducing enthalpic interactions between polymer and solute [8], despite a number of industriallyinteresting reactions take place in water-free environment. Use of other solvents different than water is considered difficult, or even not possible [9].

Attempts can be found in the open literature to correlate the rate of a given reaction to structural aspects of ion-exchange resins. In some works, the internal distribution and concentration of sulfonic groups or the reactants accessibility towards them were studied to assess the catalytic activity [10-12], or else they discerned between the activity of internal and external sulfonic acid groups [13]. Other works analyzed the swelling properties of sulfonated ion-exchangers [14]. Finally, a number of works found some correlations between the reaction rate achieved by an ion-exchanger and its functionalization degree [1519]. For the present work, it was considered appropriate to adapt the approach of Jeřábek et al. [9], who used the ISEC determination of the swollen-state morphology of a series of cationic gel-type ionexchangers with the same acid capacity to express the observed bisphenol-A synthesis reaction rate as a sum of the contributions of individual fractions of the swollen polymer. Such contributions were expressed by means of the products of the volumes occupied by each fraction, in units of mass of the water-swollen resin, and a specific activity associated with each fraction characteristic polymer chain density $\left(0.1,0.2,0.4,0.8,1.5 \mathrm{~nm} / \mathrm{nm}^{3}\right)$.

The aim of this work is to determine the contribution of each individual fraction of swollen polymer to the overall rate of a series of given reactions, namely the syntheses of methyl tert-butyl ether, ethyl tertbutyl ether, propyl tert-butyl ether and butyl tert-butyl ether, with several ion-exchange catalysts with different physicochemical properties. 


\section{Experimental}

\subsection{Chemicals}

Methanol (max. water content $0.005 \%$ wt.), ethanol (max. water content $0.02 \%$ wt.), both supplied by Panreac (Castellar del Vallès, Spain), 1-propanol (max. water content $0.005 \%$ wt.) and 1-butanol (max. water content $0.005 \%$ wt.), both supplied by Sigma-Aldrich (Tres Cantos, Spain), and a synthetic C4 mixture as the 2-methylpropene (isobutene) source, supplied by Abelló-Linde (Barcelona, Spain), were used. The composition of the C4 mixture was: $25 \%$ wt. isobutene, $40 \%$ wt. isobutane and the remaining $35 \%$ wt. trans-2-butene.

\subsection{Catalysts}

A total of sixteen different ion-exchanger resins were used as catalyst, twelve being commercially available that were supplied by Rohm and Haas and Purolite. These ion-exchangers were Amberlyst ${ }^{\mathrm{TM}} 15$ (A-15), Amberlyst ${ }^{\mathrm{TM}} 16$ (A-16), Amberlyst ${ }^{\mathrm{TM}} 35$ (A-35), Amberlyst ${ }^{\mathrm{TM}} 36$ (A-36), Amberlyst ${ }^{\mathrm{TM}} 39$ (A39), Amberlyst ${ }^{\mathrm{TM}} 40$ (A-40), Amberlyst ${ }^{\mathrm{TM}} 46$ (A-46), Amberlyst ${ }^{\mathrm{TM}} 48$ (A-48), Amberlyst ${ }^{\mathrm{TM}} 70$ (A-70), Purolite ${ }^{\circledR}$ CT175 (CT-175), Purolite ${ }^{\circledR}$ CT252 (CT-252) and Purolite ${ }^{\circledR}$ CT275 (CT-275). Lab-made partially-sulfonated resins were also used. These ion-exchangers were 306, 406, 606 and 806 [20]. All resins were macroreticular, acidic, sulfonated polymers of styrene-divinylbenzene (PS-DVB). Their relevant characteristics are listed in Table 1.

Table 1 General characteristics of ion-exchange resins used in this work

\begin{tabular}{|c|c|c|c|c|}
\hline Catalyst & Short name & $\begin{array}{l}\text { Acid Capacity, }\left[\mathrm{H}^{+}\right]^{\mathrm{a}} \\
(\text { meq H} / \mathrm{g})\end{array}$ & $\% \mathrm{DVB}^{\mathrm{b}}$ & Sulfonation type ${ }^{c}$ \\
\hline Amberlyst 15 & A-15 & 4.81 & High & $\mathrm{C}$ \\
\hline Amberlyst 16 & A-16 & 4.80 & Medium & $\mathrm{C}$ \\
\hline Amberlyst 35 & A-35 & 5.32 & High & $\mathrm{O}$ \\
\hline Amberlyst 36 & A-36 & 5.40 & Medium & $\mathrm{O}$ \\
\hline Amberlyst 39 & A-39 & 5.06 & Low & $\mathrm{C}$ \\
\hline Amberlyst 40 & A- 40 & 5.01 & High & $\mathrm{O}$ \\
\hline Amberlyst 46 & A-46 & 0.87 & High & S \\
\hline Amberlyst 48 & A-48 & 5.62 & High & $\mathrm{O}$ \\
\hline Amberlyst 70 & A-70 & 2.65 & Low & $\mathrm{S} / \mathrm{Cl}$ \\
\hline Purolite CT175 & CT-175 & 4.98 & High & C \\
\hline Purolite CT252 & CT-252 & 5.40 & Medium & $\mathrm{O}$ \\
\hline Purolite CT275 & CT-275 & 5.20 & High & $\mathrm{O}$ \\
\hline 306 & 306 & 0.81 & High & S \\
\hline 406 & 406 & 0.99 & High & $\mathrm{S}$ \\
\hline 606 & 606 & 1.89 & High & $\mathrm{P}$ \\
\hline 806 & 806 & 3.10 & High & $\mathrm{P}$ \\
\hline
\end{tabular}

${ }^{\mathrm{a}}$ Titration against standard base.

${ }^{\mathrm{b}}$ Cross-linking degree classification considered: Low (2-8\%); Medium (8-14\%); High (14-25\%).

${ }^{\mathrm{c}}$ Conventionally sulfonated (C), oversulfonated (O), partially sulfonated (P), sulfonated mainly in the microespheres surface $(\mathrm{S})$ and sulfonated/chlorinated $(\mathrm{S} / \mathrm{Cl})$.

Pretreatment of the catalysts consisted in reducing their water content, since they were supplied in wet state. Catalysts were firstly air-dried at room temperature for $48 \mathrm{~h}$ to remove the free water from the resin beads and, afterwards, introduced in an atmospheric oven during a minimum of $14 \mathrm{~h}$ at $383 \mathrm{~K}$ to remove the bounded water molecules. Catalysts were then kept in the oven until the experiment was carried out. Additionally, air-dried catalyst beans were crushed and sieved in order to obtain particle diameters ranging $0.25-0.40 \mathrm{~mm}$, that ensure no internal mass transfer influence on the overall reaction rate [21]. 


\subsection{Analysis}

Samples were taken inline from the reactor inlet and outlet streams through two sampling valves that injected $0.2 \mu \mathrm{l}$ of pressurized liquid into an Agilent gas chromatograph 7890A equipped with a capillary column (PONA Cross-linked Methyl Silicone Gum of 50 m x $0.2 \mathrm{~mm}$ x $0.5 \mu \mathrm{m}$ ). Helium (Abelló-Linde, Barcelona, Spain), with a minimum purity of $99.998 \%$, was used as the carrier gas with a flow of $0.75 \mathrm{~mL} / \mathrm{min}$. Oven temperature ranged from 308 to $343 \mathrm{~K}$ depending on the considered reaction. This system separated and quantified the reactants, the inert components of the C4 mixture, the formed ether and the possible reaction by-products.

\subsection{Apparatus and procedure}

The experimental setup consisted of a catalytic fixed bed tubular microreactor (length: $15 \mathrm{~cm}$, i.d.: $7 \mathrm{~mm}$ ) submerged in a thermostatic bath to maintain the reactor at the desired temperature. In order to get an isothermal reactor bed, catalyst was diluted with silicon carbide of the same particle size range. Silicon carbide had been proven to be inert in terms of reaction. If catalyst dilution were too large, preferential paths or by-passing effects could arise. In a previous work [21], it was found that inert/catalyst mass ratios up to 300 did not affect the kinetic results for the present system. Therefore, dilution was kept under that value for all the experiments to avoid back-mixing and channeling.

Experiments were carried out at $333 \mathrm{~K}$. Reactor feed was free of product, what means null isobutene conversion level at the reactor inlet. Alcohol/isobutene molar ratio $\left(\mathrm{R}_{\mathrm{OH} / \mathrm{B}}{ }^{\circ}\right)$ at the reactor inlet was set to 1.0. The amount of catalyst in the catalytic bed was selected to obtain low isobutene conversion level at the desired temperature in order to operate under differential regime, were reaction rate can be considered constant along the reactor, which is generally achieved when conversion values do not exceed a $10 \%$.

Firstly, only alcohol was fed and the reactor was introduced in the thermostatic bath. The aim of this procedure was to preheat the catalyst bed and to reduce, as much as possible, the remaining water in the catalyst by alcohol percolation. As seen in previous woks, when an alcohol volume of more than 10 times the catalytic bed volume is passed through the bed, it can be considered that the water content is lower than $1.6 \%$ wt. [22].

Afterwards, while the alcohol flow was kept constant, the C4 mixture was added to the feed for the reaction to proceed. Around 3-4 h were needed for each kinetic run to reach the steady state. This fact was verified by repeated chromatographic analysis at the reactor outlet.

\subsection{Calculations}

Results were quantified in terms of reaction rate with respect to isobutene, by the following expression, which applies to a plug-flow fixed bed catalytic reactor in differential conditions:

Reaction rate: $\quad-r_{I B}=\frac{F_{I B}^{o}\left(X_{I B, \text { outlet }}-X_{I B, \text { inlet }}\right)}{W_{\text {catalyst }}}$

where $F_{I B}^{\circ}$ is the isobutene reference molar flow at null conversion, $X_{I B \text {, outlet }}$ is the isobutene conversion at the reactor outlet, the inlet isobutene conversion, $X_{I B \text {, inlet }}$, had null values, and the catalyst mass, $W_{\text {catalyst, }}$ on dry basis after oven drying.

Conversion: $\quad X_{I B}=1-\frac{F_{I B, \text { outlet }}}{F_{I B, \text { inlet }}}$ 


\section{Results and discussion}

\subsection{Considered reactions}

For the present work, the alkyl tert-butyl ether syntheses from isobutene and different alcohols were selected (Fig. 1). Alkyl tert-butyl ether is formed by addition of the corresponding alcohol to isobutene (IB). In each experiment, methanol $(\mathrm{MeOH})$, ethanol (EtOH), 1-propanol (1-PrOH) or 1-butanol (1-BuOH) were used to obtain, respectively, methyl tert-butyl ether (MTBE), ethyl tert-butyl ether (ETBE), propyl tert-butyl ether (PTBE) or butyl tert-butyl ether (BTBE). They constitute a series of analogous reactions in which a progressive variation of the reaction medium properties arise, as polarity and molecular size. Furthermore, both MTBE and ETBE syntheses are major industrially-interesting chemical processes that have been widely studied, e.g. [4, 23-26].

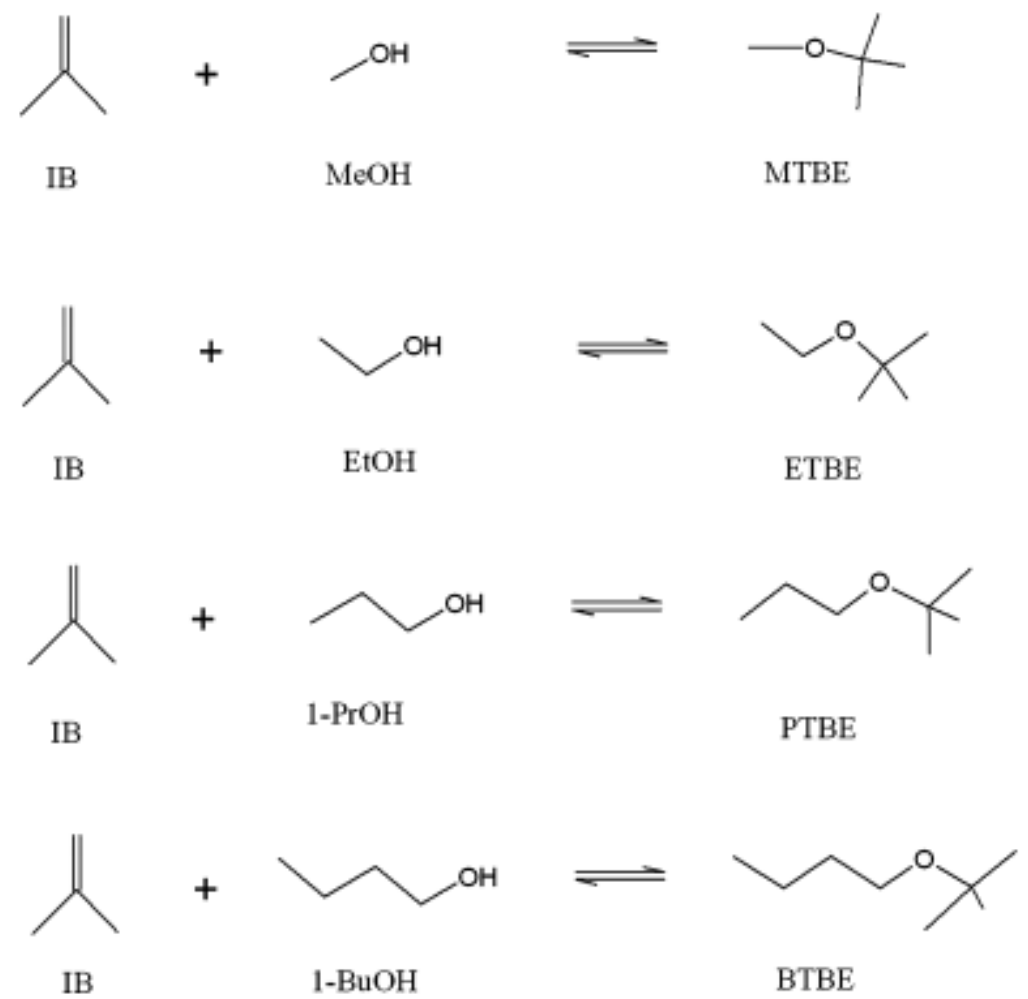

Fig.1 Main reactions involved in the present study

A simple description of this kind of reaction involves a mechanism that can be explained by a series of steps. Initially, the reactants adsorb on the resin active sites, the surface reaction between the adsorbed alcohol and isobutene follows, giving a molecule of adsorbed ether, and, finally, the ether desorbs [27]. Since the system was operated in differential regime, only forward reactions were considered to calculate rates, as under such assumption the equilibrium composition is far from being reached.

All reactions should proceed through a similar mechanism, being their main difference the size of the alcohol with which isobutene reacts. Molecule size could have implications on the adsorption extent and its penetrability into the polymer network. It can be assumed that the different polymer chain densities of a resin are a measure of the spaces available within the gel-phase. This leads to an assessment of the spatial requirements that a resin will or will not be able to fulfill for a molecule of a particular size to access the active sites.

The reactants molecular length in their minimum energy conformation, $d_{m}$, can be calculated from the distances and angles of bonds between atoms. In order to estimate such magnitudes, ChemBioOffice 2010 software was used, as it allows drawing molecules in the conformation of minimum energy and retrieve 
length, height and width of molecules. Results show that alcohols are almost linear molecules with a length ranging from $0.30 \mathrm{~nm}(\mathrm{MetOH})$ to $0.66 \mathrm{~nm}(1-\mathrm{BuOH})$, as indicated in Table 2. Isobutene is slightly bigger than ethanol.

Table 2 Molecular length, Ogston distribution coefficients, $K_{0}$, in different density domains of swollen polymer, molecular weight and random coil diameter, $\Phi_{\mathrm{d}}$, of C1-C4 linear primary alcohols and of isobutene

\begin{tabular}{|c|c|c|c|c|c|c|c|c|}
\hline \multicolumn{3}{|c|}{ Polymer chain density $\left[\mathrm{nm} / \mathrm{nm}^{3}\right]$} & 0.1 & 0.2 & 0.4 & 0.8 & 1.5 & \\
\hline \multicolumn{3}{|c|}{ Equivalent pore size $[\mathrm{nm}]^{\mathrm{a}}$} & 9.3 & 4.8 & 2.6 & 1.5 & 1.0 & \\
\hline Compound & $\begin{array}{c}\mathrm{M}_{\mathrm{w}} \\
\text { [g/mol] }\end{array}$ & $\begin{array}{l}\text { Molecular } \\
\text { length }[\mathrm{nm}]^{\mathrm{b}}\end{array}$ & & & $\mathrm{K}_{\mathrm{O}}$ & & & $\begin{array}{c}\Phi_{\mathrm{d}} \\
{[\mathrm{nm}]}\end{array}$ \\
\hline Methanol & 32.0 & 0.30 & 0.96 & 0.93 & 0.86 & 0.73 & 0.56 & 0.19 \\
\hline Ethanol & 46.1 & 0.41 & 0.95 & 0.9 & 0.81 & 0.66 & 0.46 & 0.23 \\
\hline 1-Propanol & 60.1 & 0.55 & 0.93 & 0.87 & 0.75 & 0.57 & 0.34 & 0.27 \\
\hline 1-Butanol & 74.1 & 0.66 & 0.92 & 0.84 & 0.7 & 0.49 & 0.26 & 0.31 \\
\hline Isobutene & 56.1 & 0.44 & 0.95 & 0.89 & 0.8 & 0.64 & 0.43 & 0.26 \\
\hline
\end{tabular}

${ }^{\mathrm{a}}$ From [28]

${ }^{b}$ Estimated by ChemBioOffice 2010 Software $\left(\mathrm{d}_{\mathrm{m}}\right)$

To quantify the ease of penetration of a molecule into a porous domain of a certain density, in relation to its quantity in the free solution, the Ogston distribution coefficient, $K_{O}$, estimated by [7, 28]

$$
K_{O}=\exp \left(-0.25 \pi C\left(d_{m}+d_{c}\right)^{2}\right)
$$

provides an indication of which part of a porous system is accessible to spherical molecules with a diameter $d_{m}$ in a polymer domain of a chain density $C$. Parameter $d_{c}$ stands for the diameter of the polymer chain rigid rods $\left(\mathrm{d}_{\mathrm{c}}=0.4 \mathrm{~nm}\right)$. According to Eq. $3, \mathrm{~K}_{\mathrm{O}}$ ranges from 1 to 0 , what means that either the compound amount is the same inside and outside that gel-phase zone $\left(\mathrm{K}_{\mathrm{O}}=1\right)$ or else that the compound is completely excluded from that gel-phase zone $\left(K_{O}=0\right)$. $K_{O}$ values depicted in Table 2 agree with the fact that transport through gradually denser domains is more hindered for longer alcohols than for shorter ones. It is also noticeable that $\mathrm{K}_{\mathrm{O}}$ values for a given polymer density decrease progressively as the alcohol size increases. As for the isobutene, $K_{O}$ values indicate that its mobility through the swollen-state polymer is less hindered than that of 1-PrOH.

Similarly, comparison between the random coil diameter and the equivalent pore size, of the different density domains of a swollen polymer [28], provides information on the spaces between polymer chains that a molecule would require to permeate through it without enthaplic interactions (Table 2). The random coil diameter, $\Phi_{d}$, can be estimated by the equation $[29,30]$

$\Phi_{d}=0.02457\left(\bar{M}_{w}\right)^{0.5882}$

This is an empirical measure of the space filled by a molecule of molecular weight $\bar{M}_{w}$ (g/mol). In order to ensure that a molecule can permeate without hindramce through a porous system, it is accepted that a value 2.5 times higher of $\Phi_{d}$ is needed. The equivalent pore sizes of the swollen polymer zones depicted in Table 2 are wider than this value, no matter what the molecule is, what would mean that all of the molecules considered in this study are able to permeate through the swollen-state polymer. Finally, this empirical method leads to infer that the ease of permeation through the gel-phase is ordered as methanol $>$ ethanol $>$ isobutene $>$ 1-propanol $>$ 1-butanol, the same as deduced from the Ogston distribution coefficient. 


\subsection{Catalysts characteristics}

The ion-exchangers used in the present work include polymers of low (A-39 and A-70), medium (A-16, A-36 and CT-252) and high crosslinking degree (A-15, A-35, A-40, A-46, A-48, CT-175, CT-275, 306, 406, 606 and 806). As for the sulfonation type, selected resins include conventionally sulfonated resins, with one sulfonic group per styrene ring, (A-15, A-16, A-39 and CT-175) oversulfonated resins, with more than one sulfonic group per benzene ring (A-35, A-36, A-40, A-48, CT-252 and CT-275), and partially-sulfonated resins, with a certain degree of permeation restrictions of acid sulfuric within the gel-phase during the sulfonation process (A-46, 306, 406, 606 and 806). Regarding resins A-46, 306 and 406, it has to be pointed out that local differences in sulfur concentration within the beads were detected, and therefore they are considered to be sulfonated mainly on the surface of their micropores [20]. A special feature of A-70 is that some hydrogen atoms of the polymer chain have been substituted by chlorine atoms, which improve the resin thermal stability and acid strength, leading to non-significant desulfonation by thermal stress to this resins compared to other PS-DVB resins [31].

With regards to the morphology of the gel-phase at the working-state, ISEC method provides information of the expanded volume in polymer domains of different densities swollen in water. In Figure 2, the ISEC pattern corresponding to the ion-exchangers used in this work is shown.

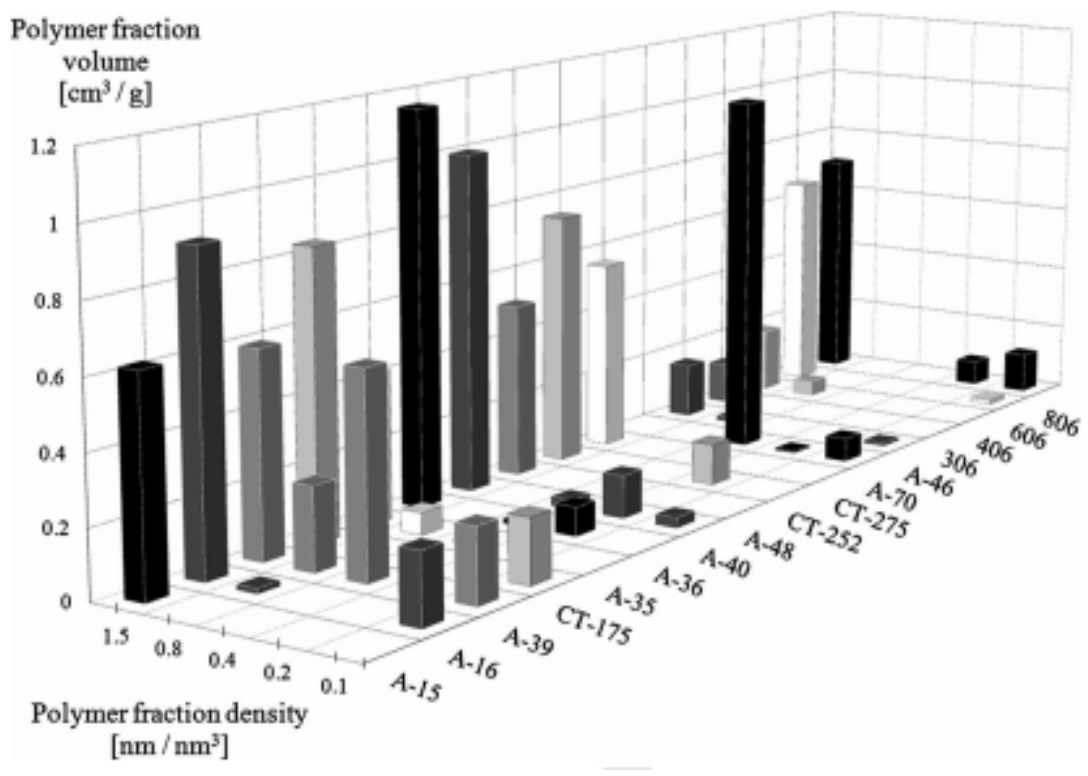

Fig.2 ISEC morphological pattern of the gel-phase

The ISEC technique employs the swollen material to be characterized, i.e. the ion-exchanger, as the stationary phase in a liquid chromatography column, and measures elution volumes of solutes with a characteristic molecular size. Then, under the considered assumptions: (a) a simple geometric model can describe the pore system, and (b) the pore system can be treated as a discrete set of fractions with simple pores of a single size, the mathematical treatment of the data provided by the ISEC analysis provides information on pore volume distributions [6, 7, 32].

It can be considered that the number of acid centers contained in a polymer mass fraction is proportional to its polymer chain length, thus to its characteristic chain density [9]. Figure 2 indicates that, in the swollen state, denser polymer fractions are generally more frequent. Actually, the densest volume $(1.5 \mathrm{~nm}$ $/ \mathrm{nm}^{3}$ ) is present in all but one of the catalysts (A-70 constitutes the sole exception) and it occupies the highest percentage of the total swollen-state volume. In case of sterically demanding reactions, the acid centers contained within the densest polymer domains become less accessible, and consequently less efficient, than the more expandable ones due to permeation hindrance. Nevertheless, their contribution to the catalytic phenomenon might be higher than those of low-density domains, because of the larger 
number of active sites due to their higher density. Also, medium and high density fractions might allow interaction between reactants and the polymer matrix.

Although the ion-exchange catalysts tailoring is a discipline yet to develop, it is obvious that information on the polymer domains efficiency for a given reaction scheme might help manufacturers to develop high efficient custom-design catalysts.

\subsection{Catalytic test}

Table 3 summarizes the catalytic performance of each ion-exchanger in the four considered reactions. Intrinsic reaction rate values depicted generally show that isobutene reacted faster with longer alcohols, and that A-35 and 306 correspond to the most and the least active resins, respectively, in the four considered reactions. This fact can be related to the catalysts functionalization degree, as A-35 presented one of the highest acid capacities and 306 presented the lowest one. Activities of all other resins do not follow a clear trend with their acid capacity values. As for the reproducibility of the experimental results presented in Table 3, notice that, although not all the experiments were replicated, the uncertainties associated to experimentally determined rates range from 0.5 to $7 \%$, which is an acceptable level of experimental error.

Table 3 Intrinsic reaction rates for alkyl tert-butyl ethers. Standard error for replicated experiments is presented. $\mathrm{T}=333 \mathrm{~K}, \mathrm{R}_{\mathrm{OH} / \mathrm{B}}^{\mathrm{o}}=1.0, \mathrm{~d}_{\mathrm{p}}=0.25-0.40 \mathrm{~mm}$, WHSV $>500 \mathrm{~h}^{-1}$

\begin{tabular}{ccccc}
\multicolumn{5}{c}{$-\mathrm{r}_{\text {IB }}\left[\mathrm{mole} /\left(\mathrm{g}_{\text {cat }} \cdot \mathrm{h}\right)\right]$} \\
\hline Catalyst & MTBE & ETBE & PTBE & BTBE \\
\hline A-35 & $0.135 \pm 0.002$ & $0.1624 \pm 0.0002$ & $0.53 \pm 0.01$ & 1.23 \\
A-15 & $0.098 \pm 0.002$ & 0.117 & 0.275 & \\
A-36 & 0.136 & 0.140 & & \\
A-16 & $0.095 \pm 0.002$ & 0.081 & 0.211 & 0.46 \\
A-39 & $0.0766 \pm 0.0004$ & $0.057 \pm 0.004$ & $0.113 \pm 0.004$ & $0.19 \pm 0.01$ \\
A-40 & 0.109 & 0.126 & & \\
A-46 & $0.0076 \pm 0.0005$ & 0.006 & 0.008 & 0.02 \\
A-48 & 0.135 & 0.152 & & \\
A-70 & 0.039 & 0.024 & 0.041 & 0.06 \\
CT-175 & 0.101 & 0.126 & & \\
CT-252 & 0.114 & 0.118 & & \\
CT-275 & 0.125 & 0.133 & $0.379 \pm 0.008$ & $0.79 \pm 0.01$ \\
306 & 0.006 & 0.005 & 0.006 & 0.01 \\
406 & 0.008 & 0.006 & 0.009 & 0.02 \\
606 & $0.0198 \pm 0.0001$ & 0.016 & 0.033 & 0.06 \\
806 & 0.043 & 0.038 & 0.098 & 0.20
\end{tabular}

From Table 3, catalysts can be classified in two groups regarding their activity progression as the size of the alcohol grows. The reactivity of primary alcohols with isobutene followed the order $n$-butanol $>n$ propanol > ethanol > methanol for resins A-35, A-15, A-36, A-40, A-48, CT-175, CT-252, and CT-275. This is in agreement with literature data on the same reaction systems with A-15 [15]. However, for resins A-16, A-39, A-46, A-70, 306, 406, 606, and 806, this order was $n$-butanol $>n$-propanol $>$ methanol $>$ ethanol.

Insight of these two groups of catalyst properties reveals that the first one was formed mainly by highly cross-linked, oversulfonated catalysts. Three exceptions can be found, though, since A-15 and CT-175 are conventionally sulfonated resins, and CT-252 is a medium cross-linked catalyst. With regards to the 
second group of catalysts, none of them was oversulfonated and they were of low, medium, and high cross-linking degree. Relative amounts of the lowest density polymer domains are generally higher for this second group of resins than for the first one, as shown in Figure 2. Such phenomenological assessment will be used in this study as a classification for the resins.

\subsection{Relations between swollen-state morphology and catalytic activity}

The role of the swelling phenomena constitutes an important feature related to the molecular permeation through the polymer backbone of a resin and the adsorption on an active site. These processes take place mainly within the swollen polymer, or gel-like phase [2]. It is then of common sense Transesterification of triacetin with methanol on solid acid and base catalysts trying to correlate the catalytic performance of a resin with its swollen-state morphology, as well as with its functionalization degree.

Because of the polar and non-aqueous environment of these reactions, it has been widely accepted that ion-exchangers experience the swelling phenomenon, but also that this swelling does not reach the degree exhibited when submerged in water. It can be assumed that each of the five specific volumes of polymer detected by ISEC will maintain their identity even though the reaction is performed in a somehow less polar organic environment [9]. Then, the ISEC information cannot be directly applicable. Nevertheless, it can also be assumed that the fraction of volume occupied by each polymer fraction in water will have a proportional relation to that occupied in organic media.

Due to the different chain density of the polymer fractions, the overall intrinsic reaction rate achieved by a catalyst can be expressed as a sum of contributions of the individual polymer domains, similar to what is proposed in a previous work [9]. For each catalyst, the individual contribution of each polymer domain of a characteristic chain density was computed as a product of a specific catalytic activity, the catalyst acidity, and the fraction of volume that it occupies, as follows

$$
r_{n}=\sum_{i=0.1}^{1.5} r_{i}=\sum_{i=0.1}^{1.5}\left[\operatorname{TOF}_{i} \cdot\left[H^{+}\right]_{n} \cdot\left(\frac{V_{S P, i}}{\sum_{j=0.1}^{1.5} V_{S P, j}}\right)\right]
$$

where: $r_{n}$ : intensive reaction rate for the catalyst $n\left(\right.$ mole $\left./\left(\mathrm{g}_{\text {cat }} \cdot \mathrm{h}\right)\right)$

$r_{i}$ : contribution to the reaction rate induced by polymer fraction $i\left(\right.$ mole $\left./\left(g_{\text {cat }} \cdot h\right)\right)$

$\mathrm{TOF}_{i}$ : specific activity of the polymer fraction $i\left(\right.$ mole $/\left(\right.$ meq $\left.\mathrm{H}^{+} \cdot \mathrm{h}\right)$ )

$\left[\mathrm{H}^{+}\right]_{n}$ : acid capacity of the catalyst $n$ (meqH $\left.{ }^{+} / \mathrm{g}_{\text {cat }}\right)$

$V_{S P, i}$ : volume occupied by fraction $i$ in the water-swollen resin $n$ per gram of catalyst $\left(\mathrm{cm}^{3} / \mathrm{g}_{\text {cat }}\right)$

$V_{S P, j}$ : volume occupied by all the gel-like fractions $j$ in the water-swollen resin $n$ per gram of catalyst $\left(\mathrm{cm}^{3} / \mathrm{g}_{\mathrm{cat}}\right)$

Specific activity, or turnover frequencies (TOF), are considered to be one of the best ways to compare catalysts on a rational basis and are often found in literature [33]. TOF can be evaluated as the quotient between reaction rate and acidity under the assumption that all active sites are accessible to reactants. In this work, the specific activity of the polymer fraction $i, T O F_{i}$, was considered to depend on the characteristic chain density. By defining $T O F_{i}$, active sites located in different polymer domains are distinguishable and can be compared in terms of their effectiveness, what can be related to the accessibility of reactants towards them.

As discussed above, catalysts can be classified into two groups according to their activity in relation with the alcohol size. Therefore, Eq. 5 was applied separately to both groups. Henceforward, resins the 
reaction rate of which followed the order $n$-butanol $>n$-propanol $>$ ethanol $>$ methanol will be referred to as group A, whereas those with an order order $n$-butanol $>n$-propanol $>$ methanol $>$ ethanol, as group B. With this procedure, two sets of up to 8 equations (one per catalyst) and two sets of 5 common unknown parameters (corresponding to the specific activity of each polymer fraction, which is equal for catalysts of a same group) are considered. Unknown parameters values were estimated by an optimization mathematical procedure in which the sum of squares of differences between experimental and calculated reaction rate values was minimized.

\subsubsection{MTBE experiments}

As Figure 3 shows, MTBE rates calculated by means of Eq. 5 fit globally well with experimental values, irrespectively of the group of considered catalysts. Residuals of the fitting presented a random distribution versus calculated reaction rate.

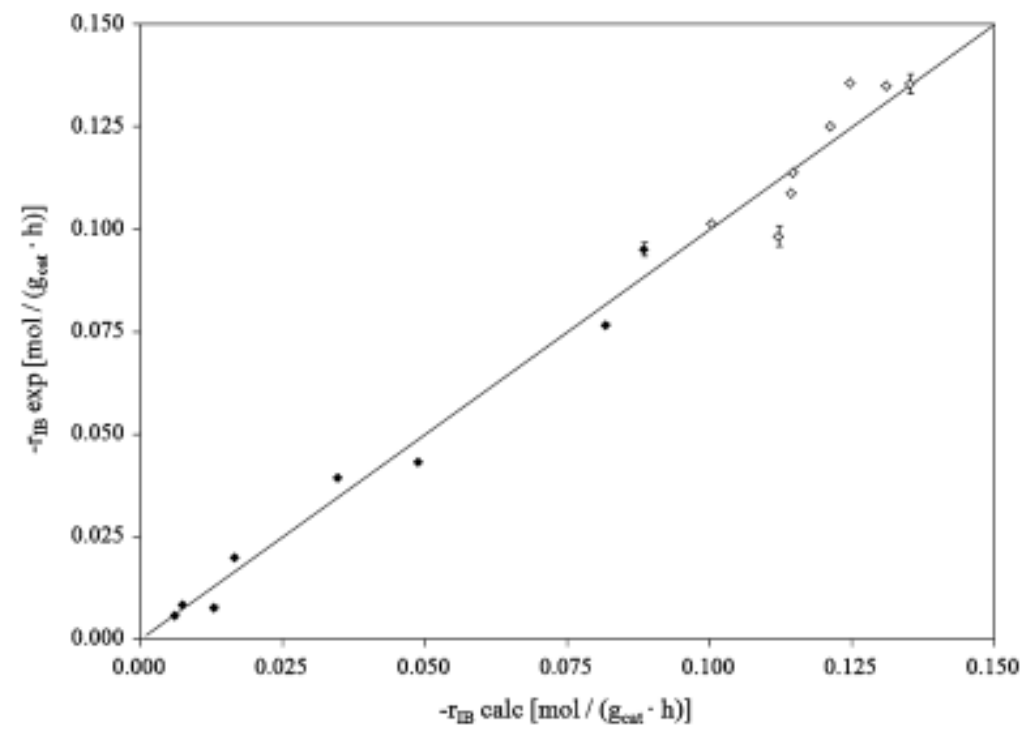

Fig.3 Reaction rate fit for the 16 catalysts in the MTBE reaction. Standard error for replicated experiments is represented with error bars. $\mathrm{T}=333 \mathrm{~K}, \mathrm{R}_{\mathrm{OH} / \mathrm{IB}}^{\mathrm{O}}=1.0, \mathrm{~d}_{\mathrm{p}}=0.25-0.40 \mathrm{~mm}$, WHSV $>500 \mathrm{~h}^{-1}$. Group A (open symbols), Group B (solid symbols)

Distribution of the computed specific activity values for each characteristic polymer chain density, $T O F_{i}$, is presented in Figure 4. As Figure 4 indicates, differences were detected regarding $T O F_{i}$ that have morphological implications. According to this model, a chain concentration of $0.4 \mathrm{~nm} / \mathrm{nm}^{3}$ provides maximum TOF in group A resins, which would be the polymer fraction working with a higher performance. On the other hand, the highest-performance polymer fraction for group B resins would be that of $0.1 \mathrm{~nm} / \mathrm{nm}^{3}$, which corresponds to the most expandable polymer domain. 


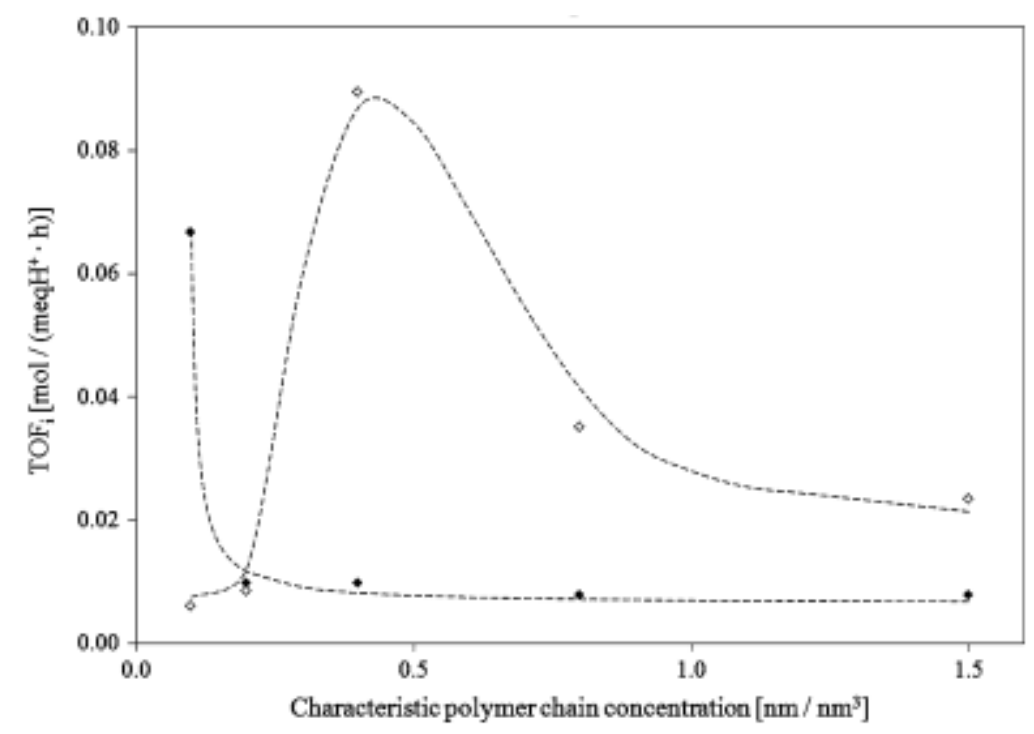

Fig.4 TOF distribution for each characteristic polymer chain density for the MTBE experiments.

$\mathrm{T}=333 \mathrm{~K}, \mathrm{R}_{\text {OH/IB }}^{\mathrm{o}}=1.0, \mathrm{~d}_{\mathrm{p}}=0.25-0.40 \mathrm{~mm}$, WHSV $>500 \mathrm{~h}^{-1}$. Group A (open symbols), Group B (solid symbols)

$\mathrm{TOF}_{\mathrm{i}}$ distribution for the MTBE experiments shown in Figure 4 should be, at first, handled with some precautions as they are necessarily bound to experimental error. Differences of $\mathrm{TOF}_{\mathrm{i}}$ could be, then, nonsignificant. However, results in Figure 4 can be explained in terms of morphological differences between resins and the catalytic activity displayed by them.

Regarding group A resins, $\mathrm{TOF}_{\mathrm{i}}$ distribution in Figure 4 suggests that active sites located in a polymer volume fraction with a characteristic chain density of $0.4 \mathrm{~nm} / \mathrm{nm}^{3}$ are the most effective. This can be related to local spatial distribution of active sites within the gel-phase that could allow coordination to multiple active sites. Participation of several groups can stabilize better the reaction intermediate. On the other hand, as low density domains present very dispersed functional groups, reactants cannot adsorb in more than one active site therein at the same time. Thicker polymer fractions $\left(>0.8 \mathrm{~nm} / \mathrm{nm}^{3}\right)$ would hinder reactants accessibility towards active sites, leading a decrease of the specific activity. According to the gel-phase distribution displayed by group A catalysts (Fig. 2), domains with $0.4 \mathrm{~nm} / \mathrm{nm}^{3}$ density represent an actually low fraction of polymer volume. Although in these domains the specific activity might be higher than the rest, their contribution to the overall reaction rate is low.

In relation to group B resins, only the lowest density polymer zone $\left(0.1 \mathrm{~nm} / \mathrm{nm}^{3}\right)$ displayed a good specific activity, whereas any density increase leads to a dramatic loss of the active sites effectiveness. In this case, rather than interpreting the results on the basis of an extremely high efficiency of active sites located in the most expandable domain, it seems that they provide wide channels that facilitate the access to denser volume fractions surrounding them. Similar inferences can be found in previous works [9]. The similarly low specific activity values displayed by polymer masses of $0.2,0.4,0.8$, and $1.5 \mathrm{~nm} / \mathrm{nm}^{3}$ nominal chain densities may be interpreted as null significant differences between their active sites effectiveness.

\subsubsection{Generalization of the empirical model}

The same procedure was applied to the ETBE experimental data, yielding similar results to those from MTBE data. Figures 5 and 6 prove this fact. 


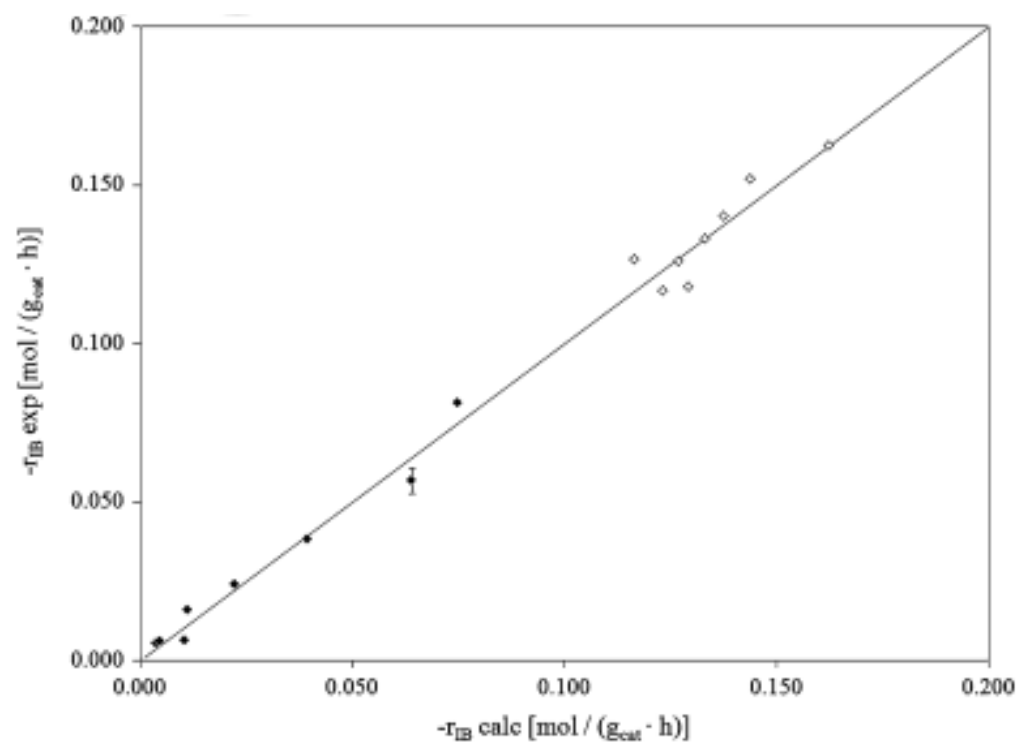

Fig.5 Reaction rate fit for the 16 catalysts in the ETBE reaction. Standard error for replicated experiments is represented with error bars. $\mathrm{T}=333 \mathrm{~K}, \mathrm{R}_{\text {OH/B }}^{\circ}=1.0, \mathrm{~d}_{\mathrm{p}}=0.25-0.40 \mathrm{~mm}$, WHSV $>500 \mathrm{~h}^{-1}$. Group A (open symbols), Group B (solid symbols)

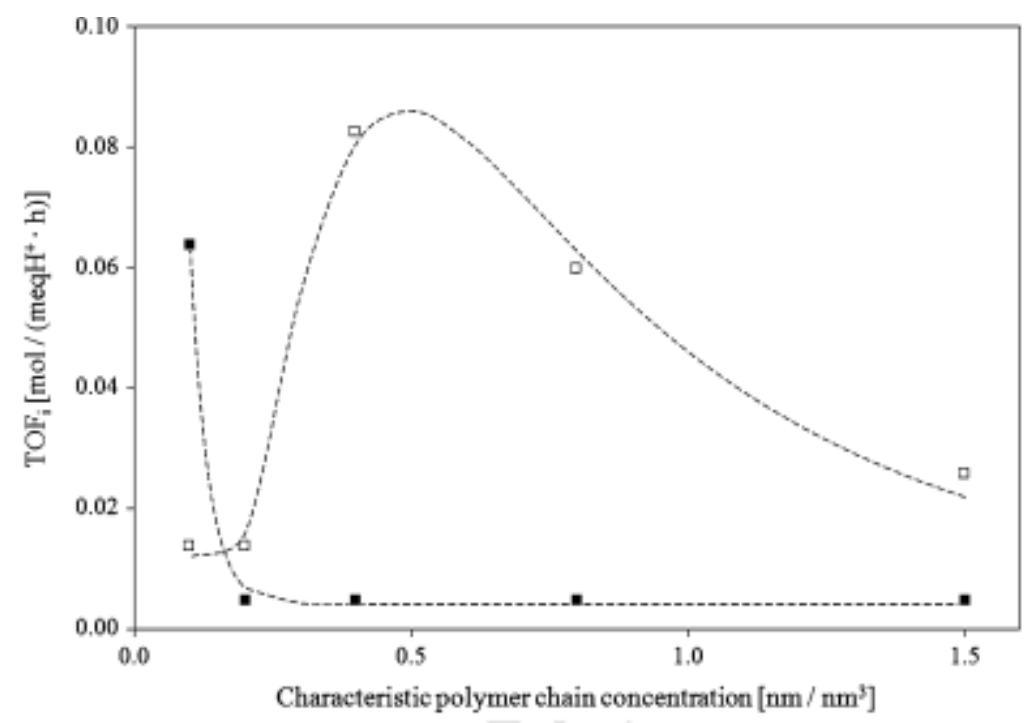

Fig.6 TOF distribution for each characteristic polymer chain density for the ETBE experiments. $\mathrm{T}=333 \mathrm{~K}, \mathrm{R}_{\mathrm{OH} / \mathrm{B}}^{\mathrm{o}}=1.0, \mathrm{~d}_{\mathrm{p}}=0.25-0.40 \mathrm{~mm}$, WHSV $>500 \mathrm{~h}^{-1}$. Group A (open symbols), Group B (solid symbols)

Consequently, it was considered appropriate to develop a generalization of the empirical equation (Eq. 5) that could be applied to similar reaction schemes. Among the Eq. 5 parameters, both acidity and polymer volume fraction distribution are specific of each studied resins Therefore, $T O F_{i}$ are the only parameters with different values for each reaction. As previously discussed, the main difference between the four considered reaction systems was the alcohol molecular length, which is inherently related to its ease of penetrability through progressively denser polymer fractions, assessed by means of the Ogston distribution coefficient (Table 2).

For such purpose, the parameter $T O F_{i}^{R}$, corresponding to volume fraction $i$ for reaction $R$, was defined relative to the MTBE formation reaction according to Eq. 6. 
$\mathrm{TOF}_{i}^{R}=k_{R} \mathrm{TOF}_{i}^{M T B E} \frac{K_{O, i}^{\mathrm{MetOH}}}{K_{O, i}^{R O H}}$

where $\mathrm{TOF}_{i}{ }^{R}$ is the specific activity of the polymer fraction $i$ for the reaction $\mathrm{R}\left(\right.$ mole / (meqH $\mathrm{H}^{+} \cdot \mathrm{h}$ )), $k_{R}$ is the proportionality constant for the reaction $\mathrm{R}, \mathrm{TOF}_{\mathrm{i}}^{\mathrm{MTBE}}$ is the specific activity of the polymer fraction $i$ for MTBE (mole / $\left(\mathrm{meqH}^{+} \cdot \mathrm{h}\right)$ ), $\mathrm{K}_{O, i}^{\mathrm{MetOH}}$ is the Ogston distribution coefficient of methanol through a polymer fraction $i$, and $K_{O, i}^{R O H}$ is the Ogston distribution coefficient of a primary linear alcohol through a polymer fraction $i$.

The parameter $T O F_{i}^{R}$ was considered to be proportional to the $T O F_{i}^{M T B E}$ and the relation between the Ogston distribution coefficient of a polymer domain, $K_{O, i}$, with methanol and the corresponding one with the considered alcohol. The relation between alcohols Ogston distribution coefficients stands for the relative differences in concentration of these compounds inside the gel-phase, in order to take into consideration the possible partial exclusion of a reactant from a particular polymer zone.

By means of this procedure, 21 equations with 5 parameters related to the polymer domain and 3 proportionality constants related to each reaction other than MTBE synthesis were considered, for group A catalysts. Regarding group B, 32 equations can be gathered with the same number and nature of unknown parameters as in group A. In total, therefore, 53 equations and 16 unknown parameters were considered. Estimation of parameters values proceeded similarly as in the MTBE case.

Figure 7 shows the results of the simultaneous fit of equations 4 and 5 to all experimental values.

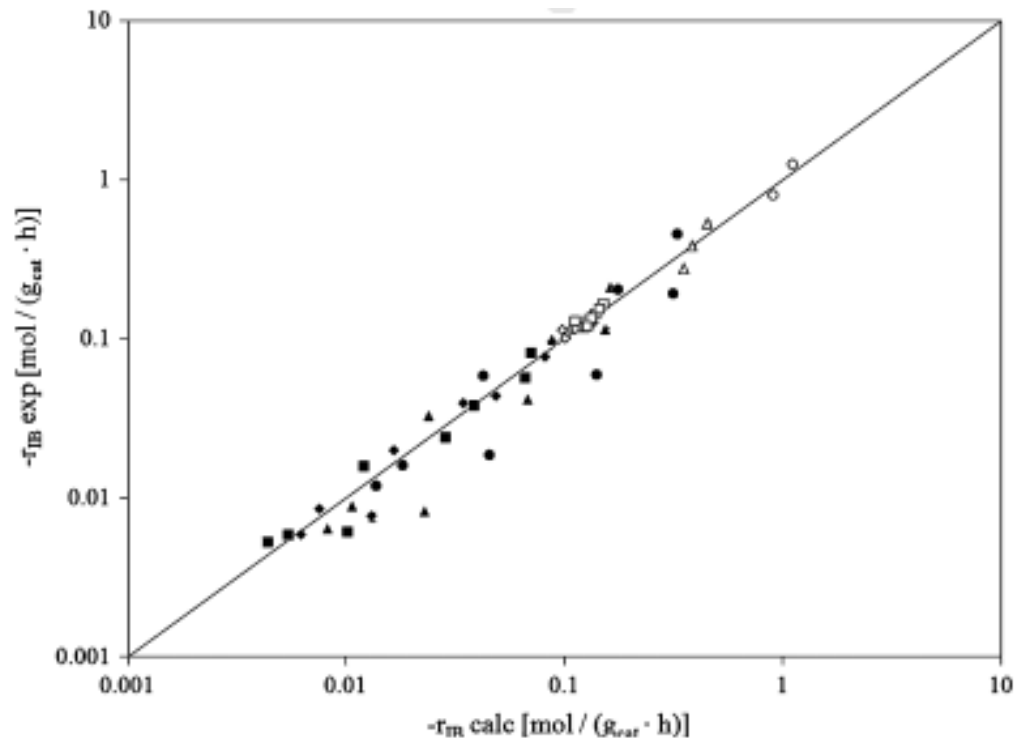

Fig. 7 Reaction rate fit for the four reactions considered. Standard error for replicated experiments is represented with error bars. $\mathrm{T}=333 \mathrm{~K}, \mathrm{R}_{\mathrm{OH} / \mathrm{B}}^{\circ}=1.0, \mathrm{~d}_{\mathrm{p}}=0.25-0.40 \mathrm{~mm}$, WHSV $>500 \mathrm{~h}^{-1}$. Catalysts: Group A (open symbols) and Group B (solid symbols). Ethers: MTBE (diamond), ETBE (square), PTBE (triangle) and BTBE (circle).

As indicated above, proportionality constants, $k_{R}$, related to the considered reaction, were obtained as fitting parameters. Figure 8 plots $k_{R}$ values against the molecular length of the alcohol involved in the reaction $R$, other than the MTBE synthesis. 


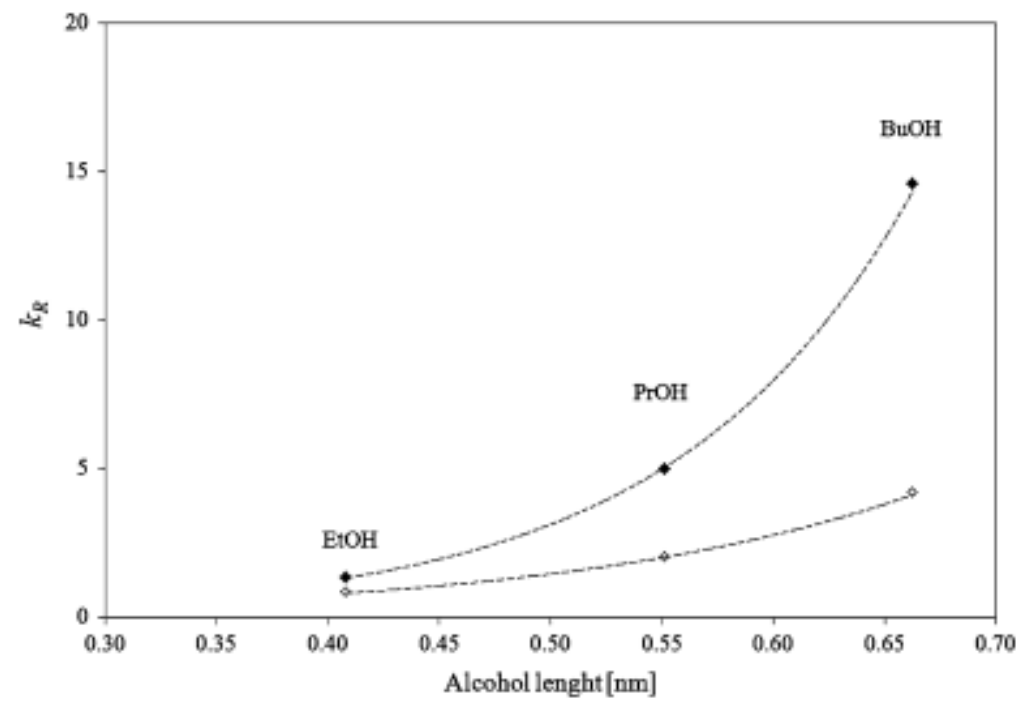

Fig. $8 k_{R}$ values versus alcohol molecular length. $\mathrm{T}=333 \mathrm{~K}, \mathrm{R}_{\mathrm{OH} / \mathrm{BB}}^{\mathrm{o}}=1.0, \mathrm{~d}_{\mathrm{p}}=0.25-0.40 \mathrm{~mm}$, WHSV $>500 \mathrm{~h}^{-1}$. Group A (open symbols), Group B (solid symbols)

As seen in Figure 8, $k_{R}$ values of both A and B resins increased exponentially with the alcohol size, which indicates that the effect of the alcohol size on the reaction kinetics is the same for each alcohol irrespectively of the catalyst type. However, relative differences between each group $k_{R}$ grew as the molecule length increased. Therefore, it can be inferred that differences between catalyst types may become more important for big molecules, which is in connection with the possible appearance of steric hindrances.

As indicated in Figure 9a and 9b, polymer fractions of 0.4 and $0.1 \mathrm{~nm} / \mathrm{nm}^{3}$ nominal chain density provide maximum specific activity for resins of groups $\mathrm{A}$ and $\mathrm{B}$, respectively. Since oversulfonated ion-exchangers would be included in group A resins, it can be stated that oversulfonated catalysts composed by a polymer mass of a characteristic chain density around $0.4 \mathrm{~nm} / \mathrm{nm}^{3}$ would be the most active resins in the etherification reactions of isobutene. 

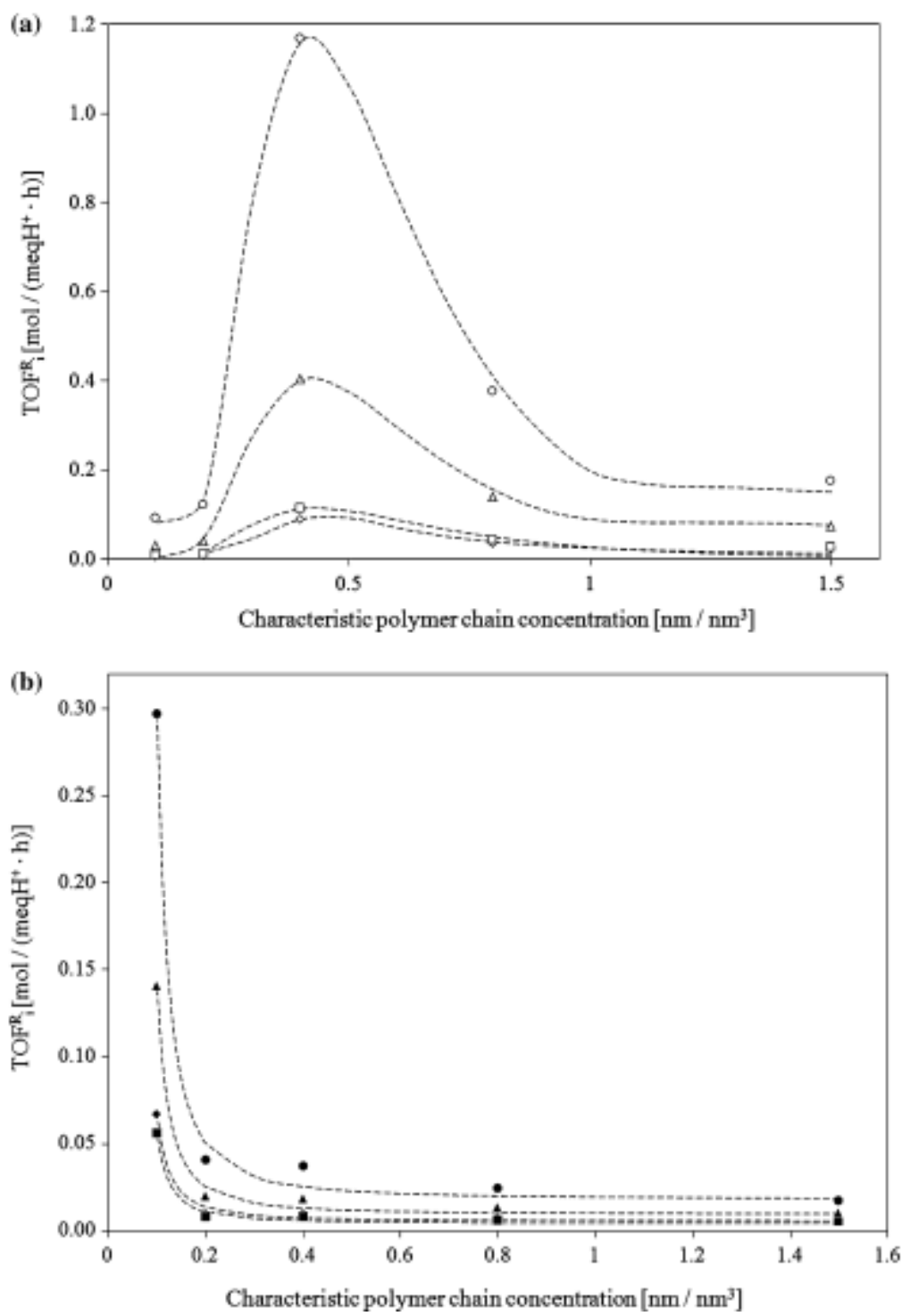

Fig. 9 TOF distribution for each characteristic polymer chain density in the four considered reactions. $\mathrm{T}=333 \mathrm{~K}, \mathrm{R}_{\mathrm{OH} / \mathrm{BB}}=1.0, \mathrm{~d}_{\mathrm{p}}=0.25-0.40 \mathrm{~mm}, \mathrm{WHSV}>500 \mathrm{~h}^{-1} . \mathrm{MTBE}$ (diamond), ETBE (square), PTBE (triangle) and BTBE (circle). A Group A catalysts (open symbols). B Group B catalysts (solid symbols)

Regarding the differences between calculated specific activities, $T O F_{i}{ }^{R}$ values depicted in Figures 9a and

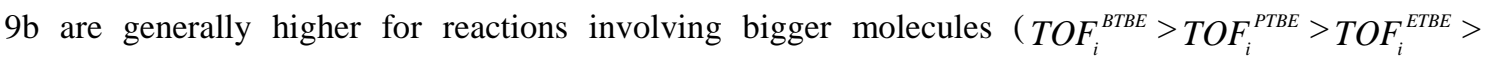
$T O F_{i}^{M T B E}$ ). These differences can be considered negligible between ETBE and MTBE reactions as well as between low $T_{O} F_{i}^{R}$ values, assuming some inaccuracy of the model and experimental error. However, a feasible interpretation of the observed differences between maximum values of specific activity in Figure 9a $\left(T O F_{0.4}^{R}\right)$ would be related to the size of the alcohol and the local concentration of active sites. Assuming both the Ogston model and the evaluation of the random coil diameter (Eq. 3 and 4), none of the considered reactants presents important permeation hindrances through the polymer. Furthermore, reactants chemisorption on active centers leads to enthalpic interactions, which can counterbalance steric hindrance that might affect molecules of similar sizes to the equivalent pore diameters of the polymer matrix [34]. Thus, within this range of molecular sizes, big molecules could coordinate to multiple active sites simultaneously easier than small molecules. This could explain the order of primary alcohols reactivity with isobutene. 
Finally, assuming this empiricism, if different catalysts need to be tested, the first thing to do would be to establish which group the catalysts belong to. Oversulfonated catalysts would probably belong to group A. Then, by comparison of their working-state morphology and acidity, an a priori estimation of their activity level could be easily achieved.

\section{Conclusions}

The empirical model presented in this work described satisfactorily experimental reaction rates for the methyl tert-butyl ether (MTBE) and the ethyl tert-butyl ether (ETBE) syntheses, separately. A generalization of the empirical model to reactions of the same nature (the propyl tert-butyl ether (PTBE), and the butyl tert-butyl ether (BTBE) syntheses) was successfully achieved by means of the Ogston distribution coefficient.

Regarding active sites effectiveness, higher specific activities were found for active sites located in polymer fractions with a characteristic chain density of $0.4 \mathrm{~nm} / \mathrm{nm}^{3}$, for a group of resins that would allow multiple active sites coordination. These resins were -35, A-15, A-36, A-40, A-48, CT-175, CT252, and CT-275. A group of resins was found that presented no favored polymer fraction, in terms of the active sites effectiveness therein. Those were A-16, A-39, A-46, A-70, 306, 406, 606, and 806. Highly acidic oversulfonated resins with a polymer fraction of a chain density around $0.4 \mathrm{~nm} / \mathrm{nm}^{3}$ would be most active catalysts for the studied reactions.

\section{Acknowledgements}

The authors thank Rohm and Haas France SAS (The Dow Chemical Company) and Purolite for providing Amberlyst and CT ion exchange resins, respectively. We are also indebted to Mrs. Júlia Athayde for her participation in part of the experimental work. Finally, the authors would like to express their deepest gratefulness to Dr. Karel Jeřábek for providing the ISEC analyses.

\section{References}

1. Bell AT (2003) The impact of nanoscience on heterogeneous catalysis. Science 299:1688-91. doi: 10.1126/science.1083671

2. Corain B, Zecca M, Jeřábek K (2001) Catalysis and polymer networks - the role of morphology and molecular accessibility. J Mol Catal A Chem 177:3-20. doi: 10.1016/S1381-1169(01)00305-3

3. Schlick S, Bortel E, Dyrek K (1996) Catalysis on polymer supports. Acta Polym 47:1-15. doi: 10.1002/actp.1996.010470101

4. Fité C, Tejero J, Iborra M, et al. (1998) The effect of the reaction medium on the kinetics of the liquidphase addition of methanol to isobutene. Appl Catal A Gen 169:165-177. doi: 10.1016/S0926860X(98)00005-2

5. Rihko-Struckmann LK, Latostenmaa P V, Krause AOI (2001) Interaction between the reaction medium and an ion-exchange resin catalyst in the etherification of isoamylenes. J Mol Catal A Chem 177:41-47. doi: 10.1016/S1381-1169(01)00308-9

6. Jeřábek K (1985) Determination of pore volume distribution from size exclusion chromatography data. Anal Chem 57:1595-1597. doi: 10.1021/ac00285a022

7. Jeřábek K (1985) Characterization of swollen polymer gels using size exclusion chromatography. Anal Chem 57:1598-1602. doi: 10.1021/ac00285a023

8. Crispin T, Halász I (1982) Determination of the pore size distribution, by exclusion chromatography, of ion-exchange polymers which swell in water. J Chromatogr A 239:351-362. doi: 10.1016/S00219673(00)81994-9 
9. Jeřábek K, Hanková L, Prokop Z, Lundquist EG (2002) Relations between morphology and catalytic activity of ion exchanger catalysts for synthesis of bisphenol A. Appl Catal A Gen 232:181-188. doi: 10.1016/S0926-860X(02)00099-6

10. AHN J (1988) The effects of the local concentration and distribution of sulfonic acid groups on 1butene isomerization catalyzed by macroporous ion-exchange resin catalysts. J Catal 113:434-443. doi: 10.1016/0021-9517(88)90269-2

11. Buttersack C (1989) Accessibility and catalytic activity of sulfonic acid ion-exchange resins in different solvents. React Polym 10:143-164. doi: 10.1016/0923-1137(89)90022-5

12. Coutinho FM., Souza RR, Gomes AS (2004) Synthesis, characterization and evaluation of sulfonic resins as catalysts. Eur Polym J 40:1525-1532. doi: 10.1016/j.eurpolymj.2004.02.003

13. Ihm SK, Chung MJ, Park KY (1988) Activity difference between the internal and external sulfonic groups of macroreticular ion-exchange resin catalysts in isobutylene hydration. Ind Eng Chem Res 27:41-45. doi: 10.1021/ie00073a009

14. Chee SY, Gan SN (2008) Swelling Properties of Copolymers of Styrene and Divinylbenzene Containing Sulfonic and Carboxylic Acid Groups. doi: 10.1002/app

15. Ancillotti F, Massi Mauri M, Pescarollo E (1977) Ion exchange resin catalyzed addition of alcohols to olefins. J Catal 46:49-57. doi: 10.1016/0021-9517(77)90134-8

16. Ancillotti F, Massi Mauri M, Pescarollo E, Romagnoni L (1978) Mechanisms in the reaction between olefins and alcohols catalyzed by ion exchange resins. J Mol Catal 4:37-48. doi: 10.1016/03045102(78)85033-0

17. Jeřábek K (1981) Polymer structure and catalytic activity of ion exchangers. Collect Czechoslov Chem Commun 46:1577-1587. doi: 10.1135/cccc19811577

18. Holub L, Jeřábek K (2005) Influence of partial neutralization on catalytic activity of ion exchange resin. J Mol Catal A Chem 231:21-26. doi: 10.1016/j.molcata.2004.12.029

19. Hanková L, Holub L, Jeřábek K (2006) Relation between functionalization degree and activity of strongly acidic polymer supported catalysts. React Funct Polym 66:592-598. doi: 10.1016/j.reactfunctpolym.2005.10.011

20. Guilera J, Hanková L, Jeřábek K, et al. (2014) Influence of the functionalization degree of acidic ionexchange resins on ethyl octyl ether formation. React Funct Polym 78:14-22. doi: 10.1016/j.reactfunctpolym.2014.02.007

21. González R (2011) Performance of Amberlyst TM 35 in the synthesis of ETBE from ethanol and C 4 cuts. 281.

22. Iborra M, Tejero J, Cunill F, et al. (2000) Drying of Acidic Macroporous Styrene-Divinylbenzene Resins with 12-20 Cross-Linking Degree. Ind Eng Chem Res 39:1416-1422. doi: 10.1021/ie9904807

23. Cunill F, Vila M, Izquierdo JF, et al. (1993) Effect of water presence on methyl tert-butyl ether and ethyl tert-butyl ether liquid-phase syntheses. Ind Eng Chem Res 32:564-569. doi: 10.1021/ie00015a020

24. Izquierdo JF, Cunill F, Vila M, et al. (1994) Equilibrium Constants for Methyl tert-Butyl Ether and Ethyl tert-Butyl Ether Liquid-Phase Syntheses using C4 Olefinic Cut. Ind Eng Chem Res 33:2830 2835. doi: 10.1021/ie00035a036 
25. Iborra M, Tejero J, El-Fassi M Ben, et al. (2002) Experimental Study of the Liquid-Phase Simultaneous Syntheses of Methyl tert- Butyl Ether (MTBE) and tert -Butyl Alcohol (TBA). Ind Eng Chem Res 41:5359-5365. doi: 10.1021/ie010640q

26. Pla R, Tejero J, Cunill F, et al. (2000) Effect of internal diffusion on liquid-phase synthesis of MTBE. 12th Int. Congr. Catal. Elsevier, pp 2609-2614

27. Tejero J, Cunill F, Izquierdo JF, et al. (1996) Scope and limitations of mechanistic inferences from kinetic studies on acidic macroporous resins The MTBE liquid-phase synthesis case. Appl Catal A Gen 134:21-36. doi: 10.1016/0926-860X(95)00191-3

28. Jeřábek K, Hanková L, Holub L (2010) Working-state morphologies of ion exchange catalysts and their influence on reaction kinetics. J Mol Catal A Chem 333:109-113. doi: 10.1016/j.molcata.2010.10.004

29. Albright RL (1986) Porous polymers as an anchor for catalysis. React Polym Ion Exch Sorbents 4:155-174. doi: 10.1016/0167-6989(86)90010-3

30. Bringué R, Ramírez E, Iborra M, et al. (2013) Influence of acid ion-exchange resins morphology in a swollen state on the synthesis of ethyl octyl ether from ethanol and 1-octanol. J Catal 304:7-21. doi: 10.1016/j.jcat.2013.03.006

31. Guilera J, Ramírez E, Fité C, et al. (2013) Thermal stability and water effect on ion-exchange resins in ethyl octyl ether production at high temperature. Appl Catal A Gen 467:301-309. doi: 10.1016/j.apcata.2013.07.024

32. Corain B, Zecca M, Jeřábek K (2001) Catalysis and polymer networks - the role of morphology and molecular accessibility. J Mol Catal A Chem 177:3-20. doi: 10.1016/S1381-1169(01)00305-3

33. López DE, Goodwin JG, Bruce D a., Lotero E (2005) Transesterification of triacetin with methanol on solid acid and base catalysts. Appl Catal A Gen 295:97-105. doi: 10.1016/j.apcata.2005.07.055

34. Jeřábek K (2013) Ion Exchanger Catalysts. Kem u Ind 62:171-176. 